\title{
Oxidant and antioxidant balance in patients with COVID-19
}

\author{
Kubra Aykac MD ${ }^{1}$ (ㅇ | Yasemin Ozsurekci MD ${ }^{2}$ () | \\ Burcu Ceylan Cura Yayla MD ${ }^{1}$ (i) | Sibel Lacinel Gurlevik MD ${ }^{2}$ (] | \\ Pembe Derin Oygar MD ${ }^{2}$ (1) | Nuriye Boduc Bolu MD ${ }^{3}$ (i) | \\ Medine Aysin Tasar MD ${ }^{3}$ (1) | Fatma Sebnem Erdinc MD ${ }^{4}$ (]) |

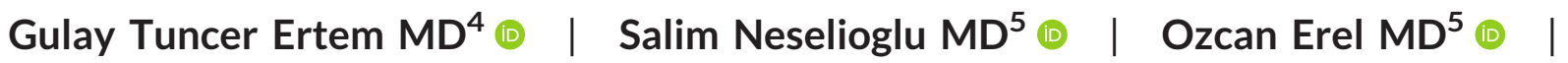 \\ Ali Bülent Cengiz MD² (1) | Mehmet Ceyhan MD $^{2}$ (])
}

\footnotetext{
${ }^{1}$ Department of Pediatric Infectious Diseases, Ankara Training and Research Hospital, University of Health Science, Ankara, Turkey

${ }^{2}$ Department of Pediatric Infectious Diseases, Hacettepe University Faculty of Medicine, Ankara, Turkey

${ }^{3}$ Department of Pediatric Diseases, Ankara Training and Research Hospital, University of Health Science, Ankara, Turkey

${ }^{4}$ Department of Infectious Diseases, Ankara Training and Research Hospital, University of Health Science, Ankara, Turkey

${ }^{5}$ Department of Clinical Biochemistry, Yildirim Beyazit University Faculty of Medicine, Ankara, Turkey

\section{Correspondence}

Kubra Aykac, Department of Pediatric Infectious Diseases, Ankara Training and Research Hospital, University of Health Science, Ankara, Turkey.

Email: kubraklnc.kk@gmail.com
}

\begin{abstract}
Background: A crucial balance exists between oxidant and antioxidant mechanisms in the functional immune system. We aimed to evaluate the contributions of balance between these systems to coronavirus disease 2019 (COVID-19), a devastating pandemic caused by viral infection.
\end{abstract}

Method: We analyzed serum oxidant and antioxidant stress parameters according to the clinical and demographic characteristics of children and adults with COVID-19 and compared them against the values of healthy controls. Serum native thiol (NT), total thiol (TT), disulfide, total antioxidant status, total oxidant status, and ischemia-modified albumin levels were evaluated and compared between groups.

Results: A total of 79 children and 74 adults were evaluated in the present study, including 46 children and 40 adults with COVID-19, 33 healthy children, and 34 healthy adults. TT, NT, and disulfide levels were significantly lower in the adult COVID-19 group than in all other groups $(p=.001, p=.001$, and $p=.005$, respectively). Additionally, TT and NT levels were significantly lower in both pediatric and adult COVID-19 cases with severe disease course than mild/moderate course. TT and NT levels were identified as predictors for the diagnosis of the adult COVID-19 cases and as independent predictors for disease severity in both children and adults with COVID-19.

Conclusion: Parameters that reveal the oxidant and antioxidant capacity, including TT and NT, appear to be good candidates for the accurate prediction of the clinical course among patients with COVID-19.

KEYWORDS

COVID-19, oxidative stress, pediatrics, severity, thiols 


\section{1 | INTRODUCTION}

The current coronavirus disease 2019 (COVID-19) pandemic is the most devastating global disease experienced in a century, and, unfortunately, its clinical manifestations, pathogenesis, and treatment remain unclear. ${ }^{1,2}$ The clinical course of this disease has ranged from asymptomatic to severe pneumonia and death. Additionally, the course of the disease differs in children and adults, and we are currently unable to predict the future prognosis of patients at the time of diagnosis. ${ }^{3}$ Therefore, an in-depth understanding of the disease pathogenesis of the disease is necessary to predict the occurrence of severe pneumonia, identify potentially critical patients, develop appropriate treatments, and reduce hospitalization rates and mortality.

Oxidative stress may be a key player in COVID-19 pathogenesis because it plays a significant role in the response to infections. ${ }^{4}$ Studies have demonstrated that oxidative stress regulates the host immune system in viral diseases, such as hepatitis B, hepatitis C, herpes simplex virus, and influenza. ${ }^{5-8}$ Additionally, oxidative stress can contribute to the pathogenesis of several lung diseases in children, such as pneumonia, asthma, acute bronchiolitis, cystic fibrosis, acute respiratory distress syndrome, and chronic neonatal lung disease. ${ }^{9-11}$ Reviews have reported a potential link between oxidative stress and the pathogenesis, severity, and mortality risk of patients infected with severe acute respiratory syndrome coronavirus (SASCoV). ${ }^{4,12,13}$ However, to the best of our knowledge, no clinical study has been performed in children with COVID-19 to investigate the oxidant and antioxidant stress parameters.

Several antioxidants can be measured in laboratories. Thiols are the main element of antioxidant defense system and good indicator of the cellular redox. ${ }^{14}$ Additionally, thiols participate in cellular proliferation, apoptosis inflammation and immune response. ${ }^{15}$ Total antioxidant status (TAS) and total oxidant status (TOS) are used to estimate the overall antioxidant status and oxidation state of the body. ${ }^{16,17}$ Another marker is ischemia-modified albumin (IMA) that has great potantial as a biomarker of oxidative stres and pneumonia. ${ }^{18,19}$

Because we suspected a potential relationship between oxidative stress and COVID-19, in this study, we evaluated the oxidant and oxidative stress statuses in both children and adults with COVID-19 compared with health controls and examined the use of oxidative stress parameters as a predictor of disease severity.

\section{2 | MATERIALS AND METHODS}

We conducted a prospective cohort study including patients diagnosed with COVID-19 between April 10, 2020, and August 10, 2020, who were admitted to University of Health Sciences, Ankara Educating and Training Hospital, and Hacettepe University Ihsan Dogramaci Children's Hospital. The study was approved by the Public Health Agency, Turkish Ministry of Health and Ethics Board of University of Health Sciences, Ankara Educating and Training
Hospital, Turkey (E-322). All parents or guardians provided written informed consent.

We diagnosed cases via reverse transcriptase-polymerase chain reaction, according to the national COVID-19 guidelines established by the Coronavirus Scientific Advisory Board in Turkey in response to new data regarding the disease ${ }^{20}$. The demographic and clinical characteristics of patients were obtained from medical records maintained by the two hospitals. We categorized the severity of pediatric COVID-19 cases based on the clinical characteristics and the results of laboratory examinations and radiologic imaging according to the highest severity during admission, as defined by Dong et al. $^{3}$

The severity of adult cases was defined according to the criteria found in the World Health Organization interim guidelines. ${ }^{21}$ At the time of admission, serum samples were obtained from pediatric and adult cases diagnosed with COVID-19 within $24 \mathrm{~h}$, and all samples were stored at $-80^{\circ} \mathrm{C}$ until oxidative stress parameters were measured. Age and gender-matched pediatric and adult healthy volunteers were enrolled as controls. Serum native thiol (NT), total thiol (TT), disulfide (DD), TAS, TOS, and IMA levels were evaluated in both patients and controls and compared between groups.

The analysis of thiol/DD levels was performed as described by Erel and Neselioglu, using a method based on the reduction of DD bonds into reactive thiol groups in the presence of $\mathrm{NaBH}_{4}{ }^{22}$ Serum TAS and TOS levels were measured using automated colorimetric measurement methods, as described by Erel. ${ }^{16,17}$ Serum IMA levels were measured using a colorimetric assay previously described by Bar-Or et al. $^{23}$

\subsection{Statistical analyses}

All statistical analyses were performed using SPSS, version 19.0 (SPSS, Inc.), and a trial version of Medcalc statistical software. Descriptive statistics were used to summarize the participants' baseline characteristics, as the median and interquartile range (IQR) for continuous variables and as frequency distributions for categorical variables. The student's $t$ test was used to compare continuous variables with a normal distribution, whereas the Mann-Whitney $U$ test was applied to comparisons of nonnormally distributed variables. Cut-off values used to distinguish between the case and control groups were determined using receiver operator characteristic (ROC) analysis, after which the sensitivity and specificity were calculated. Two-tailed tests were used for all analyses, and $p<.05$ was considered significant.

\section{3 | RESULTS}

Serum samples were collected from a total of 46 children (median age: 10 years; IQR: $3.7-14$ years; $41.3 \%$ male) and 40 adults (median age: 50 years; IQR: $43-64$ years; $52.5 \%$ male) who were diagnosed with COVID-19. The age- and gender-matched control group 
TABLE 1 Demographic, clinical, and laboratory parameters of patients with COVID-19 and healthy controls

\begin{tabular}{|c|c|c|c|c|c|}
\hline Characteristics & $\begin{array}{l}\text { Group } 1 \text { (Children with } \\
\text { COVID-19) }(n=46)\end{array}$ & $\begin{array}{l}\text { Group } 2 \text { (Adults with } \\
\text { COVID-19) }(n=40)\end{array}$ & $\begin{array}{l}\text { Group } 3 \text { (Healthy } \\
\text { children) }(n=33)\end{array}$ & $\begin{array}{l}\text { Group } 4 \text { (Healthy } \\
\text { adults) (34) }\end{array}$ & $p$ Value \\
\hline $\begin{array}{l}\text { Age (years) } \\
\quad \text { (median, Q1-Q3) }\end{array}$ & $10(3.7-14)$ & $50(43-64)$ & $9(1.5-12)$ & $41.5(39-55)$ & $\begin{array}{l}.14^{\mathrm{a}} \\
.08^{\mathrm{b}}\end{array}$ \\
\hline Male $n(\%)$ & $19(41.3)$ & $21(52.5)$ & $16(48.4)$ & $16(47.1)$ & .78 \\
\hline Severity $n$ (\%) & & & & & .02 \\
\hline Moderate & 19 (41.3) & $16(40)$ & & & \\
\hline Severe/critical & $10(21.7)$ & $18(45)$ & & & \\
\hline $\begin{array}{l}\text { Native thiol } \mu \mathrm{mol} / \mathrm{L} \\
\quad \text { (median, Q1-Q3) }\end{array}$ & $440(396-474)$ & $332(278-376)$ & $403(322-453)$ & $394(331-418)$ & $\begin{array}{l}.001^{c} \\
.43^{a} \\
.12^{b}\end{array}$ \\
\hline $\begin{array}{l}\text { Disulphide } \mu \mathrm{mol} / \mathrm{L} \\
\quad \text { (median, Q1-Q3) }\end{array}$ & $20.5(18-23)$ & $18.5(15-21)$ & $19.5(17.7-21.7)$ & $20(17.3-22.5)$ & $\begin{array}{l}.005^{c} \\
.54^{a} \\
.69^{b}\end{array}$ \\
\hline $\begin{array}{l}\text { TAS mmol Trolox } \\
\text { equiv./L, } \\
\text { (median, Q1-Q3) }\end{array}$ & $0.94(0.85-1)$ & $0.98(0.8-1)$ & $0.89(0.8-0.9)$ & $1(0.88-1.1)$ & $\begin{array}{l}.36^{c} \\
.13^{a} \\
>.05^{c}\end{array}$ \\
\hline
\end{tabular}

Note: Bold values statistically significant at $p<0.05$.

Abbreviations: COVID-19, coronavirus disease 2019; IMA, ischemic-modified albumin; TAS, total antioxidant stress; TOS, total oxidant stress.

${ }^{\mathrm{a} G r o u p ~} 1$ vs. Group 3.

bGroup 2 vs Group 4.

'Group 1 vs Group 2.

consisted of 33 healthy children and 34 healthy adults. Based on disease status, the patients were divided into 4 groups: Group 1, children with COVID-19; Group 2, adults with COVID-19; Group 3, healthy children; and Group 4, healthy adults. In Group 1, 10 (21.7\%) children had severe/critical disease (4 severe, 6 critical) and 36 (78.3\%) children had mild/moderate disease. In Group 2, 18 (45\%) adults had severe disease and 22 (55\%) adults had mild/moderate disease. Two pediatric cases and no adult cases resulted in fatalities. The pediatric patients who died were aged 2 and 7 years, and neither had any preexisting comorbidities.
When the parameters of pediatric and adult patients with COVID-19 were compared, significantly reduced serum levels of NT, TT, and DD were detected in the adult group $(p=.001, p=.001$, and $p=.005$, respectively). No significant differences were in NT, TT, and DD levels were observed for any other 2-group comparisons (Group 1 vs. Group 3 or Group 2 vs. Group 4). No significant differences in TAS, TOS, or IMA values were observed between adult and pediatric COVID-19 patients (Group 1 vs. Group 2). The demographic, clinical, and laboratory parameters of patients with COVID-19 are shown in Table 1. 
TABLE 2 Comparisons of the serum thiols, disulphide, TAS, TOS, and IMA levels of the children with COVID-19 according to the disease severity

\begin{tabular}{|c|c|c|c|c|c|}
\hline $\begin{array}{l}\text { Parameters } \\
\text { (median, Q1-Q3) }\end{array}$ & Mild cases $(n=17)$ & $\begin{array}{l}\text { Moderate } \\
\text { cases }(n=19)\end{array}$ & $\begin{array}{l}\text { Severe/critical } \\
\text { cases }(n=10)\end{array}$ & $\begin{array}{l}\text { Control } \\
\text { cases }(n=33)\end{array}$ & $p$ Value \\
\hline $\begin{array}{c}\text { Native thiol } \\
\mu \mathrm{mol} / \mathrm{L}\end{array}$ & 449 (416-475) & $445(416-511)$ & $318(276-419)$ & 403 (329-403) & $\begin{array}{l}.006^{a} \\
.002^{b} \\
>.05^{c}\end{array}$ \\
\hline Total thiol $\mu \mathrm{mol} / \mathrm{L}$ & $494(455-494)$ & $492(451-555)$ & $355(308-461)$ & $439(365-497)$ & $\begin{array}{l}.006^{\mathrm{a}} \\
.003^{\mathrm{b}} \\
>.05^{\mathrm{c}}\end{array}$ \\
\hline Disulphide $\mu \mathrm{mol} / \mathrm{L}$ & $20.5(20-23)$ & $21(19-23)$ & $17.5(16.2-22.7)$ & $19.5(17.7-21.7)$ & $>.05^{\mathrm{a}, \mathrm{b}, \mathrm{c}}$ \\
\hline $\begin{array}{l}\text { TAS mmol Trolox } \\
\text { equiv./L }\end{array}$ & $0.8(0.8-0.9)$ & $0.9(0.9-1)$ & $0.9(0.6-1.1)$ & $0.8(0.8-0.9)$ & $>.05^{\mathrm{a}, \mathrm{b}, \mathrm{c}}$ \\
\hline
\end{tabular}

Note: Bold values statistically significant at $p<0.05$.

Abbreviations: COVID-19, coronavirus disease 2019; IMA, ischemic-modified albumin; TAS, total antioxidant stress; TOS, total oxidant stress.

a Mild vs. severe/critical.

${ }^{\mathrm{b}}$ Moderate vs. severe/critical.

${ }^{\mathrm{c}}$ Control vs. severe/critical.

TABLE 3 Comparisons of the serum thiols, disulphide, TAS, TOS and IMA levels of adults with COVID-19 according to the disease severity

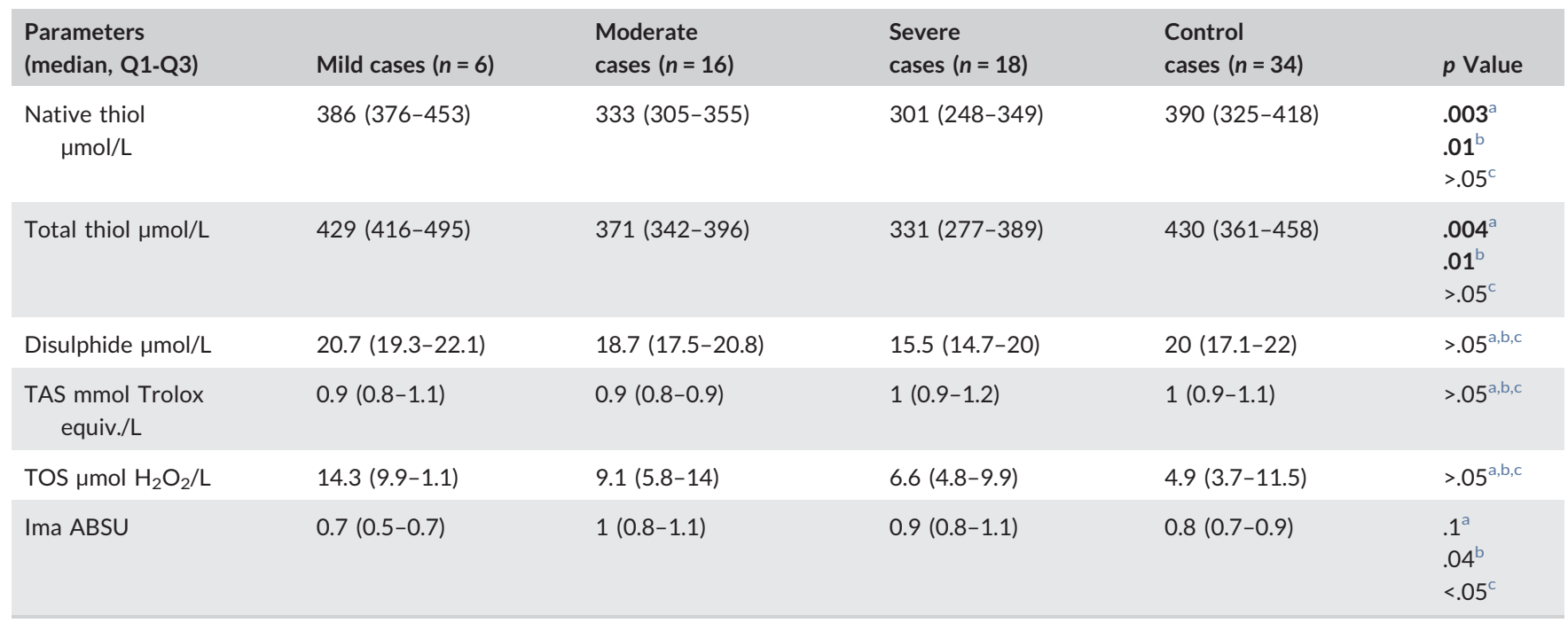

Note: Bold values statistically significant at $p<0.05$.

Abbreviations: COVID-19, coronavirus disease 2019; IMA, ischemic-modified albumin; TAS, total antioxidant stress; TOS, total oxidant stress.

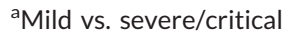

bModerate vs. severe/critical

${ }^{\mathrm{c} C o n t r o l ~ v s . ~ s e v e r e / c r i t i c a l ~}$ 
(A)

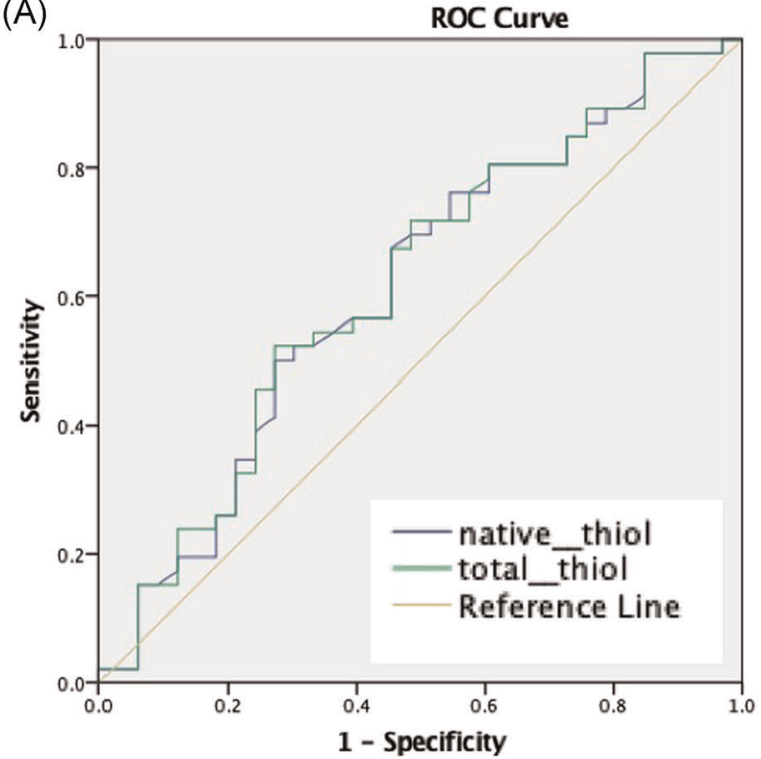

(C)

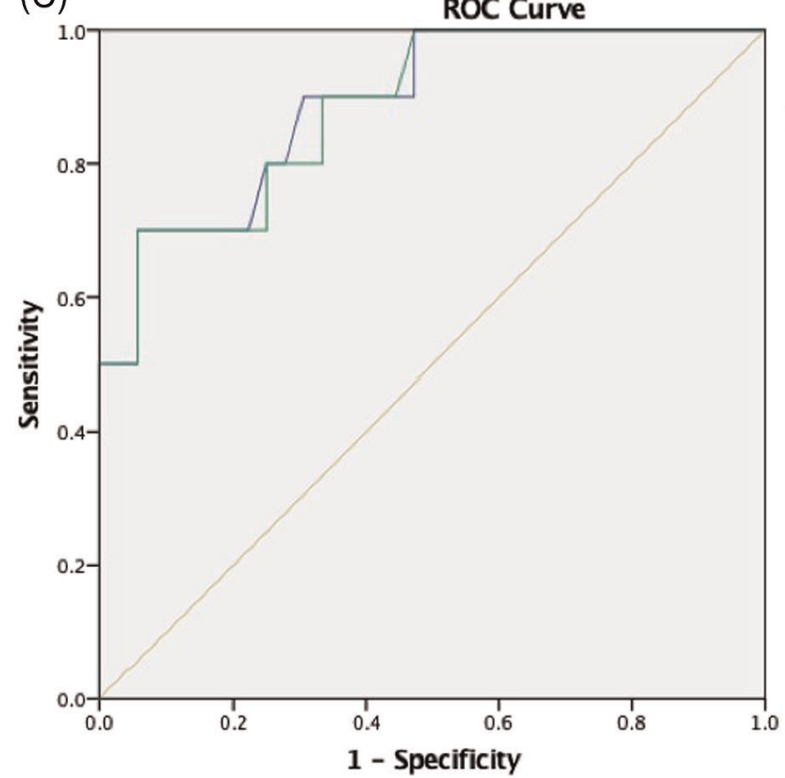

(B)

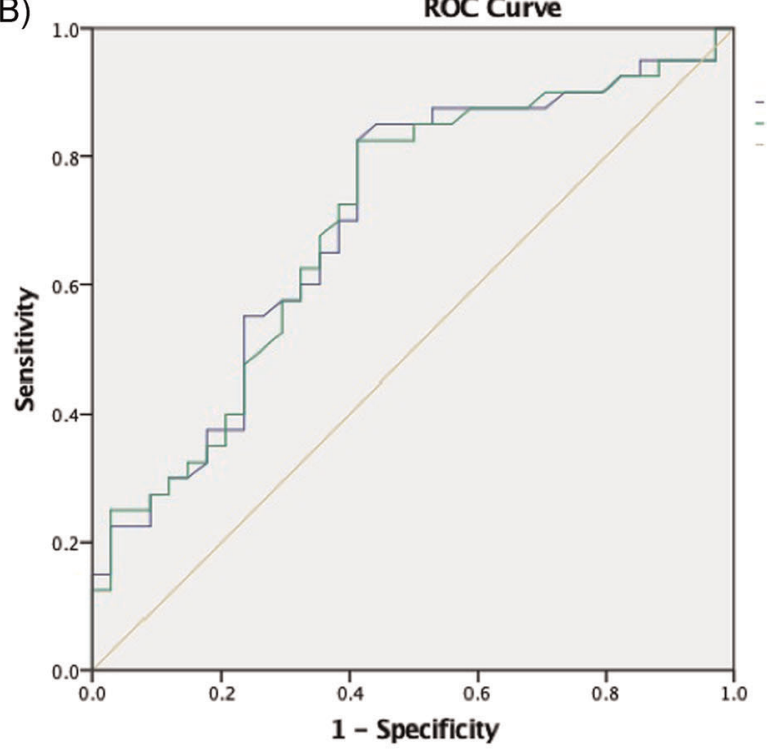

(D)

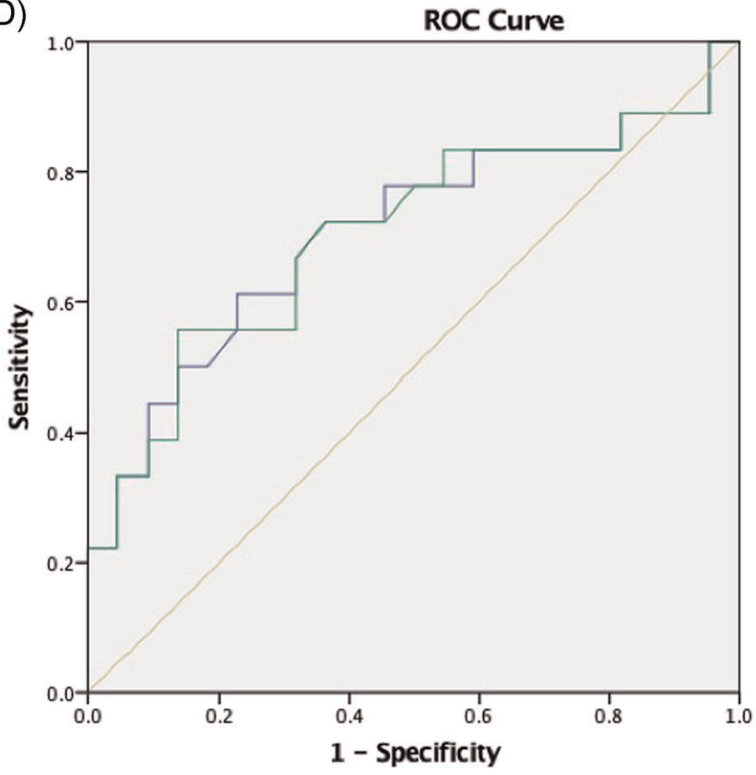

FIGURE 1 The ROC curve of serum native/total thiol levels on admission for both pediatric and adult patients with COVID-19 and pediatric and adult cases with severe disease course or not. (A) The AUC of the ROC curve for serum native/total thiol levels was calculated in children. (B) The AUC of the ROC curve for serum native/total thiol levels was calculated in adults. (C) The AUC of the ROC curve for serum native/total thiol levels was calculated in children with severe disease. (D) The AUC of the ROC curve for serum native/total thiol levels was calculated in adults with severe disease. AUC, area under the ROC curve; COVID-19, coronavirus disease 2019; ROC, receiver operator characteristic [Color figure can be viewed at wileyonlinelibrary.com]

When the parameters of pediatric patients with COVID-19 and healthy children were evaluated according to disease course, significantly lower serum TT and NT levels were observed in children with a severe/critical disease presentation compared with those with a mild or moderate disease presentation $(p=.006, p=.003$ and $p=.006, p=.002$, respectively) (Table 2 ). Consistently, significantly lower serum TT and NT levels were also observed in adults with a severe disease course compared with patients who had a mild or moderate disease course $(p=.004, p=.01$, and $p=.003, p=.01$, respectively, Table 3).

The calculated cut-off values for $\Pi \mathrm{T}$ and NT used to distinguish pediatric patients with COVID-19 were 440 and $480 \mu \mathrm{mol} / \mathrm{L}$, respectively, which resulted in sensitivities of $50 \%$ and $52 \%$ and specificities of $72 \%$ and $72 \%$. The area under the ROC curve (AUC) values (with $95 \%$ confidence intervals $[95 \% \mathrm{CI}])$ were $0.614(0.486-0.742)$ for NT and $0.618(0.490-0.745)$ for $\Pi \mathrm{(} p=.08$ and $p=.07$, respectively, Figure $1 \mathrm{~A})$. 
The calculated cut-off values for $\Pi \mathrm{T}$ and NT used to distinguish adult COVID-19 patients were 379 and $420 \mu \mathrm{mol} / \mathrm{L}$, respectively, which resulted in sensitivities of $58 \%$ and $58 \%$ and specificities of $82 \%$ and $82 \%$. The AUC $(95 \% \mathrm{Cl})$ values were $0.701(0.58-0.822)$ for NT and 0.699 (0.578-0.82) for TT ( $p=.003$ and $p=.003$, respectively, Figure 1B).

We further analyzed whether these oxidant/antioxidant stress parameters could be used to predict COVID-19 disease severity. Adult and pediatric patients were separately divided into a severe group, which contained all severe and critically ill cases, and a nonsevere group, which included both mild and moderate cases. The ROC curves for $\Pi$ and NT were calculated separately for children and adults. The results for children showed that the AUC values were 0.889 for NT and 0.885 for TT $(p=.001$ and $p=.001$, respectively, Figure $1 C$ ). The results for adults showed that the AUC values were 0.707 for NT and 0.705 for $\Pi$ ( $p=.002$ and $p=.002$, respectively, Figure 1D).

\section{4 | DISCUSSION}

We found that $\mathrm{TT}$ and NT levels were significantly lower in both pediatric and adult COVID-19 cases with severe disease course in the present study. Our findings supported the potential utility of using thiol levels as a predictor of COVID-19 severity, and the higher thiol levels observed in mild or moderate cases may serve as an indirect indicator of the improved antioxidant status in lung tissues due to better control of the proinflammatory processes in the lungs. ${ }^{24}$ Additionally, our data showed that the $\Pi T$ and NT levels were lower in adults than in children with COVID-19, and these levels appeared to be good predictors of COVID19 diagnosis, particularly among adults. The better thiol/DD balance observed in children might indicate they might are better protected from the harmful effects of oxidative stress than adults. Although severe patients presented with low thiol levels in both groups, the milder clinical course that is generally observed in children might be explained by these differences in the oxidative statuses of pediatric and adult cases.

The imbalance between oxidant production and antioxidant mechanisms is referred to as oxidative stress and can result in oxidative damage caused by the peroxidation of lipids and the oxidation of DNA. ${ }^{11}$ Reactive oxygen species (ROS) are produced by activated inflammatory cells in response to infectious diseases. ${ }^{25}$ Viral infections have been found to be associated with a decreased antioxidant defense, in addition to increased neutrophil infiltration and ROS release. ${ }^{26}$ The high neutrophil to lymphocyte ratio observed in severe COVID-19 cases, ${ }^{27}$ the neutrophilic infiltration of pulmonary capillaries, and the increase in neutrophil activation, including the circulation of neutrophil extracellular traps, have all been suggested to serve as indicators of COVID-19. ${ }^{28,29}$ Thiols are major total body antioxidants that play crucial roles against ROS, protecting against damage induced by free radicals, and thiol levels decrease during the neutralization of ROS in cases of high levels of oxidative stress. ${ }^{30,31}$ Consistent with our findings, relative to those in healthy individuals, the $\mathrm{TT}$ and NT levels have been reported to be reduced during many infectious/inflammatory processes, such as acute tonsillopharyngitis, meningism, meningitis, acute appendicitis, Crimean-Congo hemorrhagic fever, and brucellosis. ${ }^{32-36}$ Moreover, in an unpublished study performed at our center, thiol levels were found to be useful as a severity predictor for community acquired pneumonia (CAP), and the high thiol levels observed in outpatients with CAP were suggested as a potential indirect indicator of improved antioxidant status in the lung tissue, indicating the status of the proinflammatory processes in the lungs. We postulate that excessive ROS production might occur in both severe pediatric and adult COVID-19 cases; however, this requires further detailed confirmation. An in-depth understanding of the critical disease course would allow for the identification of promising avenues for the investigation of the potential pathophysiological mechanisms underlying COVID-19 and for the development of reasonable COVID-19 management strategies.

Thiol groups are among the most abundant and important antioxidant molecules found in both cells and plasma and appear to serve as the primary regulators of oxidative stress. Thiols are organic compounds contained within protein structures. Thiol groups can form reversible DD bridges through the effect of oxidants to maintain dynamic thiol-DD homeostasis. ${ }^{22}$ The thiol-DD balance can be affected by oxidative stress and is crucial for viral entry, viral reactivity, and fusion into the host cell. ${ }^{37-40}$ Hati et al. investigated the molecular basis of SARS-CoV-2 cellular recognition and reported that the binding affinity was significantly impaired when the DD bonds in both angiotensin-converting enzyme II (ACE2) and the SARS-CoV/CoV-2 spike proteins were reduced to thiol groups, which indicated that the reduction or absence of oxidative stress could have significant beneficial effects during the early stage of viral infection by preventing viral protein binding with host cells.

To gain entry into human cells, a spike glycoprotein on the viral envelope of SARS-CoV-2 interacts with the ACE2 receptor, which is expressed in various human organs. Therefore, the spike glycoprotein appears to represent a promising potential target for the development of specific drug treatments and vaccines. ${ }^{41}$ Due to the urgent necessity of identifying effective anti-SARS-CoV-2 therapeutic agents, the typical drug development process may require too much time; therefore, a focus on the potential use of currently Food and Drug Administration (FDA)approved drugs might represent a reasonable therapeutic approach. Disulfiram (DSF), which is a thiol-reacting FDA-approved drug, appears to be an acceptable candidate drug that is often used over the long term by patients with chronic alcoholism. ${ }^{42,43}$ Recently, DSF was determined to serve as an inhibitor of the papain-like proteases associated with SARSCoV-1 and Middle East respiratory syndrome coronavirus. ${ }^{44}$ Finally, both DSF and its metabolites have clearly been demonstrated to penetrate into human cells to block the proteolytic functions of $3 \mathrm{CL}^{\text {pro }}$, which is a protein that is crucial for SARS-CoV-2 replication. The results of the present study indicated that thiol-mediated inflammation should be considered in severe/critically ill COVID-19 patients and that thiolreacting agents, such as DSF, might represent a reasonable therapeutic option. However, this assumption requires additional clinical confirmation.

Several limitations of this study should be noted. First, the sample sizes were relatively small; therefore, our result cannot be generalized and require confirmation in a larger cohort to reveal the actual disease nature. Second, the detailed analyses of many of the oxidant and 
antioxidant parameters mentioned in the literature could not be performed in this study or linked with the disease course. For instance, the decreased expression of the antioxidant enzyme superoxide dismutase 3 (SOD3) in the lungs of a COVID-19 patient was associated with disease severity. ${ }^{45}$ However, we believe that the findings of our study will be able to guide physicians and improve our understanding of the potential physio-pathological pathways involved in COVID-19.

In conclusion, serum oxidant and antioxidant stress parameters could change in response to disease severity in COVID-19 patients. We found that serum TT and NT levels might serve as significant predictors of disease severity in both pediatric and adult COVID-19 patients. Evaluating the thiol-DD balance, which serves as a marker of oxidative stress, appears to be essential for understanding the pathogenesis of various viral infections, including COVID-19. In addition, Parlak et al. also concluded that the thiol/DD status might represent an important variable for monitoring the treatment response in pneumonia, ${ }^{46}$ which is a serious complication of COVID-19. Our findings shed light on the disease pathogenesis, emphasizing the significance of oxidative stress and the potential use of both new and currently available therapeutic options, such as thiol-reacting agents and metabolites for the treatment of COVID-19.

\section{CONFLICT OF INTERESTS}

The authors declare that there are no conflict of interests.

\section{AUTHOR CONTRIBUTIONS}

Kubra Aykac: conceptualization (equal); data curation (equal); investigation (equal); methodology (equal); project administration (equal); writing original draft (equal); writing review \& editing (equal). yasemin ozsurekci: conceptualization (equal); investigation (equal); project administration (equal); writing original draft (equal); writing review \& editing (equal). Burcu Ceylan Cura Yayla: data curation (equal); investigation (equal); methodology (equal); resources (equal); software (equal). Sibel Lacinel Gurlevik: data curation (equal); investigation (equal); resources (equal); software (equal); validation (equal). Pembe Derin Oygar: data curation (equal); formal analysis (equal); methodology (equal); software (equal); supervision (equal). Medine Aysin Tasar: supervision (equal); validation (equal); visualization (equal). Fatma Sebnem Erdinc: supervision (equal); validation (equal); visualization (equal). Gulay Tuncer Ertem: validation (equal); visualization (equal); writing review \& editing (equal). Salim Neselioglu: methodology (equal); supervision (equal); validation (equal); visualization (equal). Ozcan Erel: methodology (equal); supervision (equal); validation (equal); visualization (equal). Ali Bülent Cengiz: supervision (equal); validation (equal); visualization (equal). Mehmet Ceyhan: supervision (equal); validation (equal); visualization (equal); writing review \& editing (equal).

\section{DATA AVAILABILITY STATEMENT}

The data that support the findings of this study are available on request from the corresponding author. The data are not publicly available due to privacy or ethical restrictions.

\section{ORCID}

Kubra Aykac (D) http://orcid.org/0000-0002-0974-4765

Yasemin Ozsurekci (D) https://orcid.org/0000-0001-8662-6909

Burcu Ceylan Cura Yayla (D) https://orcid.org/0000-0001-5153-2136

Sibel Lacinel Gurlevik (D) https://orcid.org/0000-0001-9434-3597

Pembe Derin Oygar (D) http://orcid.org/0000-0002-8612-4586

Nuriye Boduc Bolu (D) https://orcid.org/0000-0001-5748-7241

Medine Aysin Tasar (D) https://orcid.org/0000-0003-4367-725X

Fatma Sebnem Erdinc (D) https://orcid.org/0000-0003-3918-564X

Gulay Tuncer Ertem (D) https://orcid.org/0000-0001-8760-0030

Salim Neselioglu (D) https://orcid.org/0000-0002-0974-5717

Ozcan Erel (D) https://orcid.org/0000-0002-2996-3236

Ali Bülent Cengiz (D) https://orcid.org/0000-0002-2628-6954

Mehmet Ceyhan (D) https://orcid.org/0000-0002-2318-3394

\section{REFERENCES}

1. World Health Organization (WHO): Coronavirus disease (COVID19) outbreak situation. https://www.who.int/emergencies/diseases/ novel-coronavirus-2019. Accessed 29 Sep, 2020

2. Shen $\mathrm{KL}$, Yang $\mathrm{YH}$, Jiang RM, et al. Updated diagnosis, treatment and prevention of COVID-19 in children: experts' consensus statement (condensed version of the second edition). World J Pediatr. 2020;16(3):232-239. https://doi.org/10.1007/s12519-020-00362-4

3. Dong Y, Mo X, Hu Y, et al. Epidemiological characteristics of 2143 pediatric patients With 2019 coronavirus disease in China. Pediatrics. 2020;145:e20200702.

4. Delgado-Roche L, Mesta F. Oxidative stress as key player in severe acute respiratory syndrome coronavirus (SARS-CoV) infection. Arch Med Res. 2020;51(5):384-387. https://doi.org/10.1016/j.arcmed. 2020.04.019

5. Hunt NH, van Reyk DM, Fragonas JC, Jeitner TM, Goldstone SD (1994) Redox Mechanisms in T Cell Activation. In: Pasquier C, Olivier RY, Auclair C, Packer L, (eds.). Oxidative Stress, Cell Activation and Viral Infection. Molecular and Cell Biology Updates. Birkhäuser Basel. https:// doi.org/10.1007/978-3-0348-7424-3_21

6. Ha HL, Shin HJ, Feitelson MA, Yu DY. Oxidative stress and antioxidants in hepatic pathogenesis. World J Gastroenterol. 2010; 16(48):6035-6043. https://doi.org/10.3748/wjg.v16.i48.6035

7. Gonzalez-Dosal R, Horan KA, Rahbek SH, et al. HSV infection induces production of ROS, which potentiate signaling from pattern recognition receptors: role for S-glutathionylation of TRAF3 and 6. PLoS Pathog. 2011;7:1002250.e1002250

8. Gonzalez-Dosal R, Horan KA, Rahbek SH, et al. HSV infection induces production of ROS, which potentiate signaling from pattern recognition receptors: role for S-glutathionylation of TRAF3 and 6 . PLoS Pathog. 2011;7(9):1002250. https://doi.org/10.1371/journal. ppat.1002250.e1002250

9. Aykac K, Ozsurekci Y, Tanir Basaranoglu S, et al. Oxidant and antioxidant balance in children with bacteremia [published online ahead of print Sep 3, 2020]. Minerva Pediatr. 2020;10. https://doi. org/10.23736/S0026-4946.20.05748-5

10. Ciencewicki J, Trivedi S, Kleeberger SR. Oxidants and the pathogenesis of lung diseases. J Allergy Clin Immunol. 2008;122:456-470.

11. HS Park, Kim SR, Lee YC. Impact of oxidative stress on lung diseases. Respirology. 2009;14:27-38.

12. Derouiche S. Oxidative stress associated with SARS-Cov-2 (COVID19) increases the severity of the lung disease-a systematic review. J Infect Dis Epidemiol. 2020;6:121.

13. Zeliger $\mathrm{HI}$, Kahaner $\mathrm{H}$. Can the oxidative stress index predict the severity of COVID-19? EJMED. European Journal of Medical and Health Sciences. 2020;2(2):233. 
14. Rodosskaia NK, Chernousova GM. Immune system and thiols: some peculiarities of thiol exchange. Comp Immunol Microbiol Infect Dis. 2010;33(1):65-71. https://doi.org/10.1016/j.cimid.2008.08.001

15. Moriarty-Craige SE, Jones DP. Extracellular thiols and thiol/disulfide redox in metabolism. Annu Rev Nutr. 2004;24:481-509. https://doi. org/10.1146/annurev.nutr.24.012003.132208

16. Erel O. A new automated colorimetric method for measuring total oxidant status. Clin Biochem. 2005;38:1103-1111.

17. Erel O. A novel automated direct measurement method for total antioxidant capacity using a new generation, more stable ABTS radical cation. Clin Biochem. 2004;37:277-285.

18. Bolatkale $M$, Duger $M$, Ülfer $G$, et al. A novel biochemical marker for community-acquired pneumonia: ischemia-modified albumin. Am J Emerg Med. 2017;35(8):1121-1125. https://doi.org/10.1016/j.ajem. 2017.03.018

19. Roy D, Quiles J, Gaze DC, Collinson P, Kaski JC, Baxter GF. Role of reactive oxygen species on the formation of the novel diagnostic marker ischaemia modified albumin. Heart. 2006;92(1):113-114. https://doi.org/10.1136/hrt.2004.049643

20. The Coronavirus Scientific Advisory Board (Turkey). https:// covid19bilgi.saglik.gov.tr/depo/rehberler/COVID-19_Rehberi.pdf Last update: 14 April 2020.

21. World Health Organization. Clinical management of COVID-19: interim guidance, 27 May 2020. World Health Organization, 2020 https://apps.who.int/iris/handle/10665/332196 License: CC BYNC-SA 3.0 IGO.

22. Erel O, Neselioglu S. A novel and automated assay for thiol/disulphide homeostasis. Clin Biochem. 2014;47(18):326-332. https:// doi.org/10.1016/j.clinbiochem.2014.09.026

23. Bar-Or D, Lau E, Winkler JV. A novel assay for cobalt-albumin binding and its potential as a marker for myocardial ischemia-a preliminary report. J Emerg Med. 2000;19:311-315.

24. Leelarungrayub J, Borisuthibandit T, Yankai A, Boontha K. Changes in oxidative stress from tracheal aspirates sampled during chest physical therapy in hospitalized intubated infant patients with pneumonia and secretion retention. Ther Clin Risk Manag. 2016. Published 2016;12:1377-1386. https://doi.org/10.2147/TCRM. S112972

25. Gantt KR, Goldman TL, McCormick ML, et al. Oxidative responses of human and murine macrophages during phagocytosis of Leishmania chagasi. J Immunol. 2001;167(2):893-901. https://doi.org/10.4049/ jimmunol.167.2.893

26. Laforge $\mathrm{M}$, Elbim $\mathrm{C}$, Frère $\mathrm{C}$, et al. Tissue damage from neutrophilinduced oxidative stress in COVID-19. Nat Rev Immunol. 2020;20(9): 515-516. https://doi.org/10.1038/s41577-020-0407-1

27. Fu J, Kong J, Wang W, et al. The clinical implication of dynamic neutrophil to lymphocyte ratio and D-dimer in COVID-19: a retrospective study in Suzhou China. Thromb Res. 2020;192:3-8. https:// doi.org/10.1016/j.thromres.2020.05.006

28. Barnes BJ, Adrover JM, Baxter-Stoltzfus A, et al. Targeting potential drivers of COVID-19: neutrophil extracellular traps. J Exp Med. 2020;217:e20200652. https://doi.org/10.1084/jem.20200652

29. Golonka RM, Saha P, Yeoh BS, et al. Harnessing innate immunity to eliminate SARS-CoV-2 and ameliorate COVID-19 disease. Physiol Genomics. 2020;52(5):217-221. https://doi.org/10.1152/physiolgeno mics.00033.2020

30. Biswas S, Chida AS, Rahman I. Redox modifications of protein-thiols: emerging roles in cell signaling. Biochem Pharmacol. 2006;71: 551-564.

31. Gulpamuk B, Tekin K, Sonmez K, et al. The significance of thiol/ disulfide homeostasis and ischemia-modified albumin levels to assess the oxidative stress in patients with different stages of diabetes mellitus. Scand J Clin Lab Invest. 2018;78:136-142.

32. Esen R, Aslan M, Kucukoglu ME, et al. Serum paraoxonase activity, total thiols levels, and oxidative status in patients with acute brucellosis. Wien Klin Wochenschr. 2015;127:427-433.

33. Kara SS, Erel O, Demirdag TB, et al. Alteration of thiol-disulphide homeostasis in acute tonsillopharyngitis. Redox Rep. 2017;22: 205-209.

34. Aycicek A, Iscan A, Erel O, Akcali M, Selek S. Total antioxidant/ oxidant status in meningism and meningitis. Pediatr Neurol. 2006;35: 382-386.

35. Ozyazici S, Karateke F, Turan U, et al. A novel oxidative stress mediator in acute appendicitis: thiol/disulphide homeostasis. Mediators Inflamm. 2016;2016:6761050.

36. Karadag-Oncel E, Erel O, Ozsurekci Y, et al. Plasma oxidative stress and total thiol levels in Crimean-Congo hemorrhagic fever. Jpn $J$ Infect Dis. 2014;67:22-26.

37. Suhail S, Zajac J, Fossum C, et al. Role of oxidative stress on SARS-CoV (SARS) and SARS-CoV-2 (COVID-19) infection: a review. The Protein Jornal. 2020;39:644-656.

38. Markovic I, Stantchev TS, Fields KH, et al. Thiol/disulfide exchange is a prerequisite for CXCR4-tropic HIV-1 envelope-mediated T-cell fusion during viral entry. Blood. 2004;2004(103):1586-1594.

39. Lavillette D, Barbouche R, Yao Y, et al. Significant redox insensitivity of the functions of the SARS-CoV spike glycoprotein: comparison with HIV envelope. J Biol Chem. 2006;2006(281):9200-9204.

40. Hati S, Bhattacharyya S. Impact of Thiol-Disulfide Balance on the Binding of Covid-19 Spike Protein with Angiotensin-Converting Enzyme 2 Receptor. ACS Omega. 2020;5(26):16292-16298. https:// doi.org/10.1021/acsomega.0c02125

41. Ni W, Yang X, Yang D, et al. Role of angiotensin-converting enzyme 2 (ACE2) in COVID-19. Crit Care. 2020;24(1):422. https://doi.org/10. 1186/s13054-020-03120-0

42. Lobo-Galo N, Terrazas-López M, Martínez-Martínez A. FDAapproved thiol-reacting drugs that potentially bind into the SARSCoV-2 main protease, essential for viral replication. J Biomol Struct Dyn. 2020;39(9):3419-3427.

43. Yoshimura A, Kimura M, Nakayama $\mathrm{H}$, et al. Efficacy of disulfiram for the treatment of alcohol dependence assessed with a multicenter randomized controlled trial. Alcohol Clin Exp Res. 2014;38(2): 572-578. https://doi.org/10.1111/acer.12278

44. Lin MH, Moses DC, Hsieh CH, et al. Disulfiram can inhibit MERS and SARS coronavirus papain-like proteases via different modes. Antiviral Res. 2018;150:155-163. https://doi.org/10.1016/j.antiviral. 2017.12.015

45. Abouhashem AS, Singh K, Azzazy HME, Sen CK. Is low alveolar type II Cell SOD3 in the lungs of elderly linked to the observed severity of COVID-19? Antioxid Redox Signal. 2020;33(2):59-65. https://doi.org/ 10.1089/ars.2020.8111

46. Parlak ES, Alisik M, Hezer H, Karalezli A, Hasanoglu HC, Erel O. Evaluation of dynamic thiol/disulfide redox state in communityacquired pneumonia. Saudi Med J. 2018;39(5):495-499. https://doi. org/10.15537/smj.2018.5.22111

How to cite this article: Aykac K, Ozsurekci Y, Yayla BCC, et al. Oxidant and antioxidant balance in patients with COVID-19. Pediatric Pulmonology. 2021;56:2803-2810. https://doi.org/10.1002/ppul.25549 